\title{
DELAY SYSTEMS WITH MEROMORPHIC FUNCTIONS DESIGN
}

\author{
Jiř́i Korbel, Libor Pekař \& Roman Prokop
}
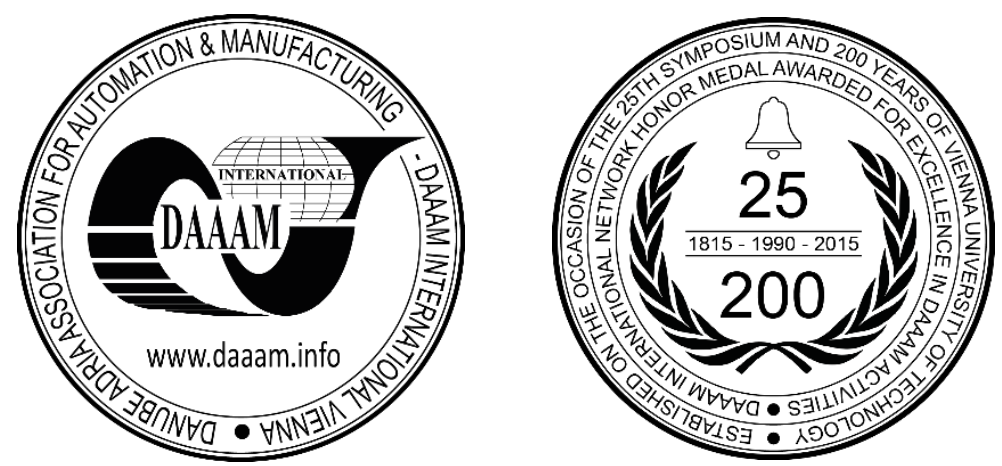

This Publication has to be referred as: Korbel, J[iri]; Pekar, L[ibor] \& Prokop, R[oman] (2017). Delay Systems with Meromorphic Functions Design, Proceedings of the 28th DAAAM International Symposium, pp.0224-0228, B. Katalinic (Ed.), Published by DAAAM International, ISBN 978-3-902734-11-2, ISSN 1726-9679, Vienna, Austria

DOI: $10.2507 / 28$ th.daaam.proceedings.030

\begin{abstract}
The contribution is focused on analysis and synthesis of delay systems in a ring of RQ-meromorphic functions. Controller design is based on solution of Diophantine equations in this ring that leads to Smith predictor like structure. Tuning of the controllers is based on pole-placement method as a desired multiple root of the characteristic closed loop equation. A stable second order transfer function is used as an example to validate the methodology. Furthermore, a biased relay experiment is combined with the proposed control design to bring an autotuning method. Matlab-Simulink examples are given to illustrate the developed approach.
\end{abstract}

Keywords: Delay systems; Diophantine equation; Meromorphic functions; Relay experiment

\section{Introduction}

Most of the real processes in the industry are time delay systems. Study and analysis of those systems became an attractive and active topic for researchers for many decades [1], [2]. Various approaches and methods were developed for compensating the time delay because it negatively affects system dynamics. Behavioral approach [3], ring of pseudopolynomials [4] or fractional representation [5], [6], [7] can be mentioned as representatives of those methods.

Automatic tuning of controllers exists in many modifications and principles. During the automatic tuning procedure, the unknown system parameters are identified and a suitable controller is designed. The first part can be achieved by performing a biased relay experiment that estimates the controlled system parameters. For the following controller design, a various methods exist.

This paper uses a combination of biased relay experiment and algebraic controller design in a ring of RQ-meromorphic functions [8]. This approach enables designing the controller for time delay systems without the need of approximations of controlled system time delay term.

\section{Delay systems}

Algebraic tools are frequently used in control theory. These tools include, among others, also rings. Control theory uses several types of rings, e.g. the ring of proper and stable rational functions $R_{P S}$, the ring of polynomials $R_{P}$ or the ring of proper and stable meromorphic functions $\mathrm{R}_{\mathrm{MS}}$. 
Zítek and Kučera [8] developed the $\mathrm{R}_{M S}$ ring for delay systems. An element of $\mathrm{R}_{\mathrm{MS}}$ is a ratio of two retarded quasipolynomials $y(\mathrm{~s}) / x(\mathrm{~s})$. A retarded quasipolynomial $x(s)$ of degree $n$ means

$$
x(s)=s^{n}+\sum_{i=0}^{n-1} \sum_{j=1}^{h} x_{i j} s^{i} \exp \left(-\vartheta_{i j} s\right), \vartheta_{i j} \geq 0
$$

Quasipolynomial (1) is stable when it owns no finite zero $s_{0}$. The publication [8] deals with stability tests. When the degree of the numerator is less or equal to the degree of the denominator the ratio $y(\mathrm{~s}) / x(\mathrm{~s})$ is proper.

A linear time-invariant delay system can be written as a ratio of two $R_{M S}$ elements. A second order system with time delay can be expressed as

$$
G(s)=\frac{\frac{b_{0} e^{-\tau s}}{\left(s+m_{0} e^{-\theta s}\right)^{2}}}{\frac{(T s+1)^{2}}{\left(s+m_{0} e^{-\theta s}\right)^{2}}}=\frac{\frac{b(s)}{m(s)}}{\frac{a(s)}{m(s)}}, \quad m_{0}>0
$$

\section{Controller synthesis}

The controlled system is estimated by second order model (2) as a ratio of two elements $B(s)$ and $A(s)$ in $R_{M S}$ ring, where each element's denominator $\Theta=0$. The system parameters $K, T$ and $\tau$ are estimated by biased relay experiment. Parameter $m_{0}>0$ is a free scalar parameter. The element $B(s)$ contains time delay term $e^{-\tau}$. The control loop is a simple feedback system with a controller $G_{R}(s)=Q(s) / P(s)$, which can be seen in 0

The control synthesis must achieve stabilization of the feedback loop, asymptotic tracking of the reference value and attenuation of the load disturbance.

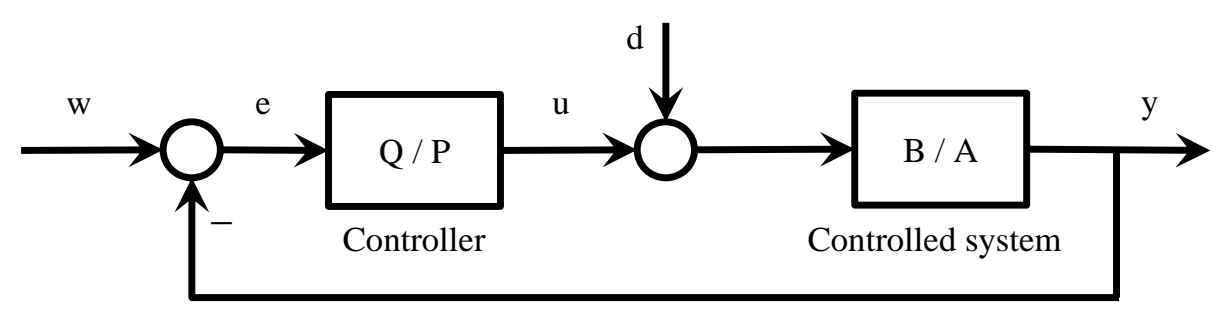

Fig. 1. Feedback control loop

Stabilization of the system can be formulated by the Diophantine equation

$$
A(s) P_{0}(s)+B(s) Q_{0}(s)=1
$$

where $P_{0}(s)$ and $Q_{0}(s)$ are particular solutions. All stabilizing controllers are derived from

$$
\frac{Q(s)}{P(s)}=\frac{Q_{0}(s)+A(s) Z(s)}{P_{0}(s)-B(s) Z(s)}, \quad P_{0}(s)-B(s) Z(s) \neq 0
$$

where $Z(s)$ is an arbitrary element of $R_{M S}$. The right choice of this element can ensure additional control conditions [5], [6], [8]. The reference and load disturbance can be described by equations

$$
W(s)=H_{W}(s) / F_{W}(s), D(s)=H_{D}(s) / F_{D}(s)
$$

Conditions for asymptotic tracking and disturbance attenuation result from expression for $E(s)$ which is

$$
E(s)=\frac{A(s) P(s)}{A(s) P(s)+B(s) Q(s)} W(s)-\frac{B(s) P(s)}{A(s) P(s)+B(s) Q(s)} D(s)
$$

It is required that $E(s)$ belongs to $R_{M S}$. It is necessary that both $F_{W}(s)$ and $F_{D}(s)$ divide $P(s)$. Details about divisibility in $R_{M S}$ and $R_{P S}$ can be found in [5], [6], [8]. Both signals $w(t)$ and $d(t)$ are step functions. Thus, for the case of $R_{P S}$ ring, it is equivalent to reach the absolute term of $P(s)$ equal to zero. The last condition is not possible to reach in $R_{M S}$, due to 
$B(s)$ and/or $P_{0}(s)$ containing delay term $\exp (-\tau s)$. For this case, the following expression for the absolute term in $P(s)$ is required

$$
\lambda\left(1-e^{-\tau s}\right)
$$

where $\lambda$ is a selected real parameter, usually $\lambda=m_{0}^{n}$, where $n$ is the order of the controlled system. Equation (4) says that the controller $G_{R}(s)$ has integral behavior for $\mathrm{s} \rightarrow 0$. This condition is assured by a proper choice of $Z(s)$ in (4). If $w(t)$ or $d(t)$ are other functions, divisibility conditions can be more complex.

For the second order system, the control design is given as follows. The equation (3) takes the form

$$
(T s+1)^{2} P_{0}(s)+K e^{-\tau s} Q_{0}(s)=\left(s+m_{0}\right)^{2}
$$

By the choice $Q_{0}(s)=1$, the solution is obtained as

$$
P_{0}(s)=\frac{\left(s+m_{0}\right)^{2}-K e^{-\tau s}}{(T s+1)^{2}}
$$

and the general solution is given by

$$
\frac{Q(s)}{P(s)}=\frac{1+\frac{(T s+1)^{2}}{\left(s+m_{0}\right)^{2}} Z(s)}{\frac{\left(s+m_{0}\right)^{2}-K e^{-\tau s}}{(T s+1)^{2}}-\frac{K e^{-\tau s}}{\left(s+m_{0}\right)^{2}} Z(s)}
$$

To obtain $P(s)$ in a simple form, let be

$$
Z(s)=\frac{\kappa\left(s+m_{0}\right)^{2}}{(T s+1)^{2}}
$$

where $\kappa$ is a real free parameter. It is clear that $Z(s) \in R_{M S}$. The choice $\kappa=\left(m_{0}^{2} / K\right)-1$ gives $P(s)$ a simple finite-dimensional form with the denominator of the degree two and

$$
P(s)=\frac{s^{2}+2 m_{0} s+m_{0}^{2}\left(1-e^{-\tau s}\right)}{(T s+1)^{2}} ; Q(s)=\frac{m_{0}^{2}}{K}
$$

Thus, the final form of the controller is

$$
G_{R}(s)=\frac{m_{0}^{2}}{K} \frac{(T s+1)^{2}}{s^{2}+2 m_{0} s+m_{0}^{2}\left(1-e^{-\tau s}\right)}
$$

where $m_{0}>0$ is a real positive tuning parameter. It is obvious that the controller is in the quasipolynomial form. The construction of this controller is more complex than usual PI or PID controllers.

\section{Biased relay feedback experiment}

The method was proposed by Åström and Hägglund. It is based on a symmetrical relay feedback test when a relay of magnitude $h_{r}$ is inserted in the feedback loop. The period of the limit cycle is the ultimate period $T_{u}$ and a limit cycle of amplitude $a_{r}$ is generated by the process output. However, there are another relays used in identification experiments. The biased (asymmetrical) relay experiment used for identification can give the final model transfer function according to [9] in the form

$$
G(s)=\frac{K e^{-\tau s}}{(T s+1)^{2}}
$$


Almost every stable industrial process can be approximated by model (14). The gain $K$ of the process can be estimated by the expression [9]

$$
K=\frac{\int_{0}^{i T_{u}} y(t) d t}{\int_{0}^{i T_{u}} u(t) d t} ; \quad i=1,2,3, \ldots
$$

and time constant $T$ is given by

$$
T=\frac{T_{u}}{2 \pi} \cdot \sqrt{\frac{4 \cdot K \cdot h_{r}}{\pi^{2} \cdot a_{r}^{2}}-1}
$$

Methodology in [10] also enables to estimate a delay term $\tau$ in (14) by the relation

$$
\tau=\frac{T_{u}}{2 \pi}\left[\pi-2 \operatorname{arctg} \frac{2 \pi T}{T_{u}}-\operatorname{arctg} \frac{\varepsilon}{\sqrt{a_{r}^{2}-\varepsilon^{2}}}\right]
$$

where $\varepsilon$ is a relay hysteresis. For the subsequent controller design estimated parameters $K, T$ and $\tau$ are used.

\section{Verification of the methodology}

Consider a stable system with time delay described by the transfer function

$$
G(s)=\frac{10}{(20 s+1)(5 s+1)(3 s+1)} \cdot e^{-10 s}
$$

Transfer functions of the type (18) represent a wide class of frequent stable industrial processes. The biased relay experiment was used to approximate the system and relations (15) - (17) give the approximation as

$$
G^{*}(s)=\frac{10}{(12.03 s+1)^{2}} \cdot e^{-10.59 s}
$$

Asymmetric relay with hysteresis was used during the experiment and following parameters were set: $h_{r}=0.225(0.25$ when off, -0.2 when on), $\varepsilon=0.05$. The comparison of step responses of systems (18) and (19) is shown in Figure 2.

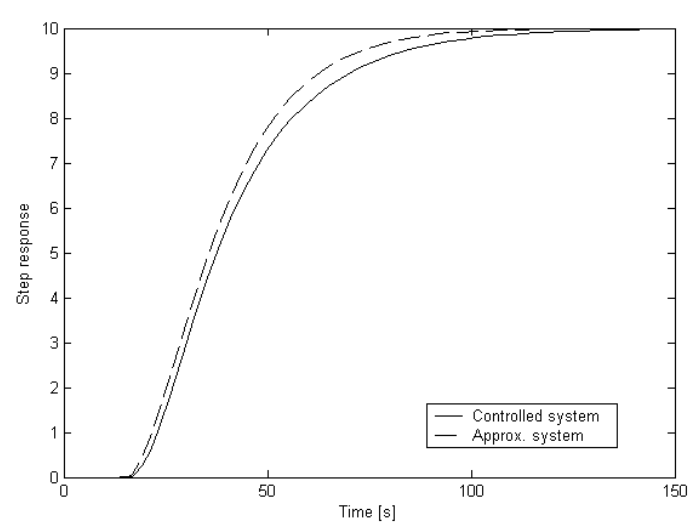

Fig. 2. Step responses of systems (18) and (19).

According to the algebraic control design in the $R_{M S}$ ring, the following controller was obtained through (8) - (13)

$$
G_{R}(s)=\frac{3.89 s^{2}+0.65 s+0.37}{100 s^{2}+10 s+0.27\left(1-e^{-10.59 s}\right)}
$$


For the comparison, another controller was also designed by the algebraic method in the $R_{P S}$ ring. Pade approximation of delay term $e^{-\tau s}$ was used. This approach gives a more general class of PID controllers. A suitable choice for $m_{0}$ was found experimentally by simulations.

Control responses are compared in Figure 3. The responses are similar, nevertheless, taking the controller (20) provides the shortest time to observe the reference value and gives the smallest "undershoot" after disturbance injection.

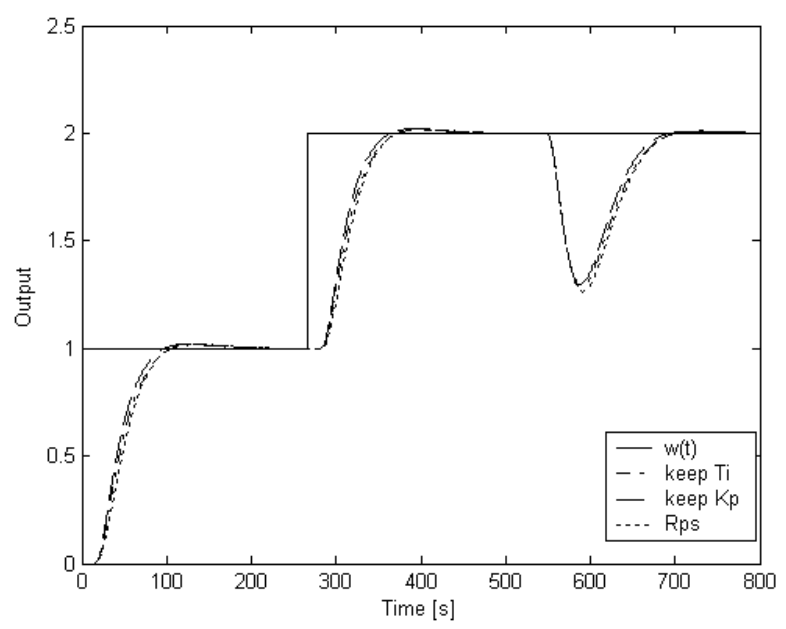

Fig. 3. Control responses for algebraic design in $R_{M S}$ and in $R_{P S}$ with Pade approximation.

\section{Conclusion}

The contribution gives a methodology for a set of delayed systems. The control synthesis was performed through a solution of a Diophantine equation in the ring of proper and stable RQ-meromorphic functions. A second order methodology generates a class of generalized Smith-like controllers with the realistic PID structure. The approach also offers a scalar tuning parameter $m_{0}>0$ which can be adjusted by a suitable principle. With a combination of biased-relay feedback identification, it brings a new autotuning method.

The developed methodology was implemented in the Matlab-Simulink environment and the approach was illustrated by an example for a higher order dynamic. It proves that the RQ-meromorphic controller is able to control the delayed systems without the need of approximation of the controlled system time delay term, like Pade approximation in $\mathrm{R}_{\mathrm{PS}}$ synthesis.

\section{References}

[1] Kamen, E. W. (1975). On the algebraic theory of systems defined by convolution operations. Mathematical Systems Theory, Vol. 9, No. 1, pp. 57-74.

[2] Morse, A. S. (1976). Ring models for delay-differential systems. Automatica, Vol. 12, No. 5, pp. $529-531$.

[3] Gluesing-Lueerssen, H. (1997). A behavioral approach to delay-differential systems. SIAM Journal on Control and Optimization, Vol. 35, No. 2, pp. 480-499.

[4] Brethé, D. \& Loiseau, J. J. (1998). An effective algorithm for finite spectrum assignment of single-input systems with delays. Mathematics and Computers in Simulation, Vol. 45, No. 4-5, pp. 339-348.

[5] Vidyasagar, M. (2011). Control system synthesis: a factorization approach, Calif.: Morgan \& Claypool, ISBN 9781608456611, San Rafael.

[6] Kučera, V. (1993). Diophantine equations in control - A survey. Automatica, Vol. 29, pp. 1361-75.

[7] Dlapa, M. (2010). Robust Controllers for Anisochronic Delayed Systems, Annals of DAAAM for 2010 \& Proceedings of the 21st International DAAAM Symposium, 20-23rd October 2010, Zadar, Croatia, ISSN 17269679, ISBN 978-3-901509-73-5, Katalinic, B. (Ed.), pp. 0075-0076, Published by DAAAM International Vienna, Vienna.

[8] Zítek, P. \& Kučera, V. (2003). Algebraic design of anisochronic controllers for time delay systems. International Journal of Control, Vol. 76, No. 16, pp. 905-921.

[9] Vítečková, M. \& Víteček, A. (2010). Plant identification by relay methods, Proceedings of Engineering the future, Sciyo, Rijeka, Dudas, L. (Ed.), pp. 242-256.

[10] Prokop, R.; Korbel, J. \& Prokopová, Z. (2010). Relay feedback autotuning - A polynomial approach, Proceedings of 24th European Conf. on modelling and Simulation, Kuala Lumpur, ISBN 978-0-9564944-1-2, pp. $290-295$. 